\title{
Jeans that fit: weighing the mass of the Milky Way analogues in the $\Lambda$ CDM universe
}

\author{
Prajwal R. Kafle, ${ }^{1 \star}$ Sanjib Sharma, ${ }^{2}$ Aaron S. G. Robotham, ${ }^{1}$ Pascal J. Elahi ${ }^{1}$ \\ and Simon P. Driver ${ }^{1}$ \\ ${ }^{1}$ ICRAR, The University of Western Australia,35 Stirling Highway, Crawley, WA 6009, Australia \\ ${ }^{2}$ SIfA, A28 School of Physics, The University of Sydney, Sydney, NSW 2006, Australia
}

Accepted 2018 January 10. Received 2018 January 10; in original form 2017 March 5

\begin{abstract}
The spherical Jeans equation is a widely used tool for dynamical study of gravitating systems in astronomy. Here, we test its efficacy in robustly weighing the mass of Milky Way analogues, given they need not be in equilibrium or even spherical. Utilizing Milky Way stellar haloes simulated in accordance with $\Lambda$ cold dark matter $(\Lambda \mathrm{CDM})$ cosmology by Bullock and Johnston and analysing them under the Jeans formalism, we recover the underlying mass distribution of the parent galaxy, within distance $r / \mathrm{kpc} \in[10,100]$, with a bias of $\sim 12$ per cent and a dispersion of $\sim 14$ per cent. Additionally, the mass profiles of triaxial dark matter haloes taken from the SURFS simulation, within scaled radius $0.2<r / r_{\max }<3$, are measured with a bias of $\sim-2.4$ percent and a dispersion of $\sim 10$ percent. The obtained dispersion is not because of Poisson noise due to small particle numbers as it is twice the later. We interpret the dispersion to be due to the inherent nature of the $\Lambda \mathrm{CDM}$ haloes, for example being aspherical and out-of-equilibrium. Hence, the dispersion obtained for stellar haloes sets a limit of about 12 per cent (after adjusting for random uncertainty) on the accuracy with which the mass profiles of the Milky Way-like galaxies can be reconstructed using the spherical Jeans equation. This limit is independent of the quantity and quality of the observational data. The reason for a non-zero bias is not clear, hence its interpretation is not obvious at this stage.
\end{abstract}

Key words: Galaxy: halo-Galaxy: kinematics and dynamics.

\section{INTRODUCTION}

A dynamical model is an essential tool for robust measurement of a mass of a galaxy. In particular, for the Milky Way (MW: Galaxy), there are a variety of techniques to measure its mass, e.g. the orbital evolution of satellite galaxies (Lin \& Lynden-Bell 1982; Besla et al. 2007); the timing argument (Li \& White 2008); the escape velocity (Smith et al. 2007; Piffl et al. 2014); directly fitting tracer kinematics and spatial properties with some parametric models (Dehnen \& Binney 1998; McMillan 2011; Irrgang et al. 2013) or distribution function (Wilkinson \& Evans 1999; Wang et al. 2015; Binney \& Piffl 2015; Eadie \& Harris 2016); or calibrating from numerical simulations (Xue et al. 2008; Rashkov et al. 2013). For a more comprehensive list of the literature that attempts to measure the dynamical mass of the MW galaxy, see review articles Courteau et al. (2014) and Bland-Hawthorn \& Gerhard (2016).

All these mass modelling schemes are known to have their own shortcomings, and a common conclusion that can be inferred from the literature is that the typical precision in the mass measurement

^E-mail: prajwal.kafle@uwa.edu.au of the Galaxy is roughly 30 percent at best (Wang et al. 2015, see Fig. 1). Generally, the systematics or biases introduced by the method of choice are largely ignored or inaccessible, and a detailed comparison of the different methods and their relative performances is the matter of a separate review. Inherently, the main challenge in our ability to achieve higher accuracy in these mass estimates is due to the lack of the tangential information of the halo tracers. With the ESA's Gaia mission (Gaia Collaboration et al. 2016), we have now entered an era of precision astrometry. The upcoming DR2 and the subsequent data releases of the mission are expected to deliver the full phase-space information of the vast number of stars (Perryman 2002; Brown, Velázquez \& Aguilar 2005) that is anticipated to allow us to measure the dynamical properties of the Galaxy, especially its mass with unprecedented accuracy.

But, the Gaia data may not be a panacea as under the current framework of hierarchical structure formation, spiral galaxies like the Galaxy are considered to be formed through the merger of multiple proto-systems. In the Galaxy, there are both qualitative and quantitative observational evidence for the presence of a plethora of unrelaxed dynamical structures, such as stellar streams and perished dwarf-galaxies (e.g. Majewski 1993; Belokurov et al. 2006; Bell et al. 2008; Helmi 2008; Starkenburg et al. 2009; Cooper et al. 


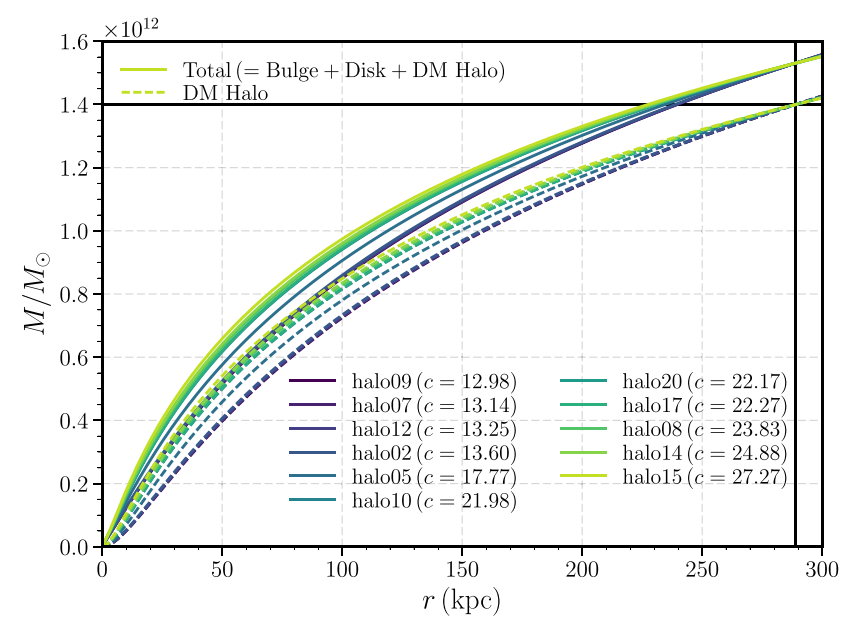

Figure 1. Mass profiles of the $11 \mathrm{BJ} 05 \Lambda \mathrm{CDM}$ haloes and the parent galaxy. Dashed lines show the DM halo mass, whereas the solid lines show the combined mass due to bulge, disc, and halo. Vertical and horizontal black solid lines correspond to the virial properties of the haloes for the concentration parameter of $c=14$. The systematic drift in the runs is due to the concentration $c$ varying among the haloes.

2011), which supports the scenario of hierarchical galaxy formation. Unrelaxed sub-structures can violate the dynamical consistency of models as well as assumptions of azimuthal symmetry and sphericity. Such features perturb the matter distribution of the host galaxy causing the gravitational field to vary with time, which further complicates the mass measurement. While fitting the galaxy whether one should include or mask these sub-structures is still open to discussion. Moreover, the Gaia data also come with uncertainties, which become even more significant at larger distances where the stellar halo is known to dominate. For example, even for RR Lyrae stars at galactocentric radius of $40 \mathrm{kpc}$, the percentage error in Gaia distance measurement is $>100$ per cent and error in the tangential velocity is of the order of atleast $50 \mathrm{~km} \mathrm{~s}^{-1}$ (Brown et al. 2005; Price-Whelan \& Johnston 2013).

An another approach for measuring the mass of the galaxy is a moment-based method using the Jeans (1915) equation, which we focus on this paper. If the first and second order moments of the velocity of the mass tracers and their stellar density distribution, which are usually observables, are known, we can use the Jeans equation to infer the gradient of the underlying gravitational potential and hence, the mass of the system. In astrophysics, the Jeans formalism has often been used for the dynamical study of gravitational systems, ranging from the galaxy superclusters (McLaughlin 1999), clusters (Carlberg et al. 1997a,b; Biviano \& Girardi 2003), groups (Faltenbacher \& Mathews 2007; Duarte \& Mamon 2015), and distant galaxies (Tonry 1983; Binney, Davies \& Illingworth 1990; Cappellari 2008) to stellar clusters (Gebhardt \& Fischer 1995; Côté et al. 2001; Wolf et al. 2010; Diakogiannis, Lewis \& Ibata 2014) and satellite galaxies (Gilmore et al. 2007; Łokas 2009; Walker et al. 2009; Battaglia, Helmi \& Breddels 2013; Diakogiannis et al. 2017). It has also been extensively used for the Galaxy (Battaglia et al. 2005; Gnedin et al. 2010; Samurović \& Lalović 2011; Kafle et al. 2012, 2014) and also recently in the neighbouring M31 galaxy (Kipper et al. 2016). Generally, a key assumption made in these analysis is that the system is in dynamical equilibrium, without this assumption no information on the underlying gravitational potential can be obtained. Furthermore, Binney (2013) argues that our strategy must be to start from the assumption of a steady state and then to use perturbation theory to understand how time-dependent effects modify a steady-state model. However, a crucial question to ask is to what extent do the unrelaxed sub-structures present in galaxies upset the estimated mass of the host using the Jeans analysis? This is the main question we aim to find answer to in this paper.

More recently there have been attempts to test the efficacy of different methods in robustly measuring the mass distributions of the MW-like galaxies using spatial and kinematic data. For example, Candlish et al. (2016) demonstrate the ability of cylindrical Jeans equation in reconstructing the surface density, the vertical force, and other disc parameters. Similarly, Wang et al. (2015) test the accuracy of a phase-space distribution function in measuring the mass of the MW dark matter halo. Besides, Sanderson, Helmi \& Hogg (2015) investigate the application of action-space clustering of tidal streams, Sanders \& Binney (2013) test stream modelling algorithm in action-angle space, and An, Evans \& Deason (2012) demonstrate the application of tracer mass formalism in correctly recovering the underlying potential of galaxies. Similarly, in this paper, we test the Jeans (1915) formalism, in particular, of its spherical form in reconstructing the mass distribution of the $\mathrm{N}$-body models of the MW.

With the advent of large spectroscopic surveys, such as Sloan Extension for Galactic Understanding and Exploration (SEGUE, Yanny et al. 2009), LAMOST Experiment for Galactic Understanding and Exploration (LEGUE, Deng et al. 2012), and GALactic Archaeology with HERMES (GALAH, De Silva et al. 2015; Martell et al. 2017), we now have a large collection of stars of different stellar types, tracing snapshots of the different age populations within the Galactic stellar halo. Battaglia et al. (2005) and Kafle et al. (2014) have combined different stellar populations, such as field horizontal branch stars, giant stars etc. to measure the mass of the Galaxy. If these different sub-populations of stars trace similar spatio-kinematics loci, or in other words they have been orbiting in the galaxy for sufficient time to become fully mixed, it is sensible to combine and treat them as a single population from a dynamical point of view. Hence, it is also instructive to understand possible bias in the mass measurement resulting from the use of different stellar populations as a tracer.

The rest of this paper is organized as follows. Section 2 describes the data sets obtained from Bullock \& Johnston (2005, hereafter BJ05) and Johnston et al. (2008, hereafter J08) simulations used in the paper. Section 3 provides the formulary for the Jeans formalism, and also, a test case is given in Section 3.2. In Section 4, we present our results and discuss them. Finally, we summarize our findings in Section 5.

\section{DATA}

Over the last decade, there has been substantial development in simulating stellar haloes in a cosmological context. Full hydrodynamical simulations of galaxies including star formation and feedback recipes have been done (Abadi, Navarro \& Steinmetz 2006; Springel et al. 2008; Zolotov et al. 2009; Vogelsberger et al. 2014; Fattahi et al. 2016; Sawala et al. 2016). However, modelling the stellar halo of galaxies is still a challenging task as it is intrinsically faint (Freeman \& Bland-Hawthorn 2002; Helmi 2008; McConnachie et al. 2009). The highest resolution simulations only resolve stellar masses of $10^{4}-10^{5} \mathrm{M}_{\odot}$, and are unable to discern the features in the stellar halo. Pushing this further to resolve the faint structures within the stellar halo would require enormous computation power. Such simulations will be further complicated by the gas inflow into 
galaxies, the star formation physics and its feedback, effects that are poorly understood. Alternatively, one could utilize models from hybrid techniques (Bullock \& Johnston 2005; De Lucia \& Helmi 2008; Cooper et al. 2010) that use collision-less simulations to track the evolution of dynamical tracers in an analytical potential, and also incorporate star formation processes in a semi-analytical form. In this paper, we use the BJ05 and J08 suites of simulations. These are readily available and have been widely used in the MW studies for its ability to reproduce sub-structures in great detail (Bell et al. 2008; Sharma \& Johnston 2009; Sharma et al. 2011a).

The BJ05 and J08 simulations adopt a two-phase approach: a simulation phase where the $N$-body simulations of the dark matter (in a short form, DM) satellites with known binding energy, accretion time, and eccentricity adopted from the $\Lambda$ cold dark matter $(\Lambda \mathrm{CDM})$ models are run in an alytic disc, bulge and halo potential representing the parent galaxy; and a prescription phase where star particles are embedded within the cores of the accreted satellite dark haloes. In other words, the satellite stellar distribution is not modelled during the $N$-body simulations, rather to each DM particle within a satellite the stars are painted on subsequently. Semi-analytical prescriptions are used to assign a star formation history to each accreted satellite, and the luminosity function of the satellite galaxies is assumed to follow King model. Afterwards, the orbit of the accreted satellites that contain a significant stellar component is tracked from entry into the parent halo up to the present time. Only satellite haloes more massive than $10^{8} \mathrm{M}_{\odot}$ are tracked as the smaller systems never contain enough number of star particles and thus do not contribute significantly to the build-up of the tracer population of the parent halo. Times since accretion, luminosity, and the eccentricity of the orbit of a satellite are the three key properties that define the course of its accretion. A full accretion history of the tracer population is a result of the combination of these three properties of all the contributing satellites.

Finally, the above hybrid simulations provide us with two types of dynamical tracers, namely (a) accreted DM particles and (b) stellar particles (essentially accreted DM particles with luminosity weights), originating from dissolving satellites. Note, the density distribution of the stellar particles shows more sub-structures than the DM particles as the stellar particles are more tightly bound to the accreting satellites. Since, the stellar particles are the ones that have the observational relevance, for our analysis, we primarily make use of them. For the sake of completeness, the results with DM tracer particles are presented in the Appendix.

\subsection{Simulated stellar haloes with $\Lambda \mathrm{CDM}$ accretion history}

The BJ05 simulation comprise a suite of 11 random realizations of the MW stellar halo, namely,

(i) haloes: 02, 05, 07, 08, 09, 10, 12, 14, 15, 17, and 2011,

here onward we collectively refer to them as $\Lambda \mathrm{CDM}$ haloes.

As we mentioned earlier, the potential of the parent galaxy is comprised of three components - Hernquist (1990) bulge, Miyamoto \& Nagai (1975) disc, and the Navarro-Frenk-White (Navarro, Frenk \& White 1996, NFW) DM halo. The concentration parameter describes the characteristic inner scaleradius of the DM halo, and evolves with time as the galaxy accretes more and more satellites.

\footnotetext{
${ }^{1}$ we keep the numbering convention of the haloes identical to http://galaxia.sourceforge.net/Galaxia3pub.html (GALAXIA, Sharma et al. 2011b), which are originally labelled as haloes 1-11 in BJ05.
}

It can be found by combining the table 1 and the eqn. 5 of BJ05, and we provide the value for each case in Fig. 1. Also, in the figure, we display the present day (redshift, $z=0$ ) masses of the parent galaxy as a function of halo-centric distance $r$, where the total combined mass due to bulge, disc, and DM halo is shown with solid lines and the sole contribution of the DM halo is shown with the dashed-lines. The horizontal and vertical black solid lines demarcate the virial mass $M_{\text {vir }}=1.4 \times 10^{12} \mathrm{M}_{\odot}$ and the virial radius $r_{\mathrm{vir}}=289 \mathrm{kpc}$, respectively, which are constants for all the simulated stellar haloes. In the figure, we see that the effect of choice of concentration on the overall mass profiles of the parent galaxy is substantial at small radii, and negligible near and past the virial radius.

It is important to note here that although the disc of the parent galaxy is assumed to have an axisymmetric potential, the underlying DM halo potential is spherically symmetric and this may be of some concern as the DM haloes under $\Lambda \mathrm{CDM}$ paradigm are expected to be of triaxial nature (e.g. Jing \& Suto 2002; Vera-Ciro et al. 2014). We discuss the cases with triaxial halo in Section 4.3.

\subsection{Simulated stellar haloes with artificial accretion history}

Besides the $\Lambda \mathrm{CDM}$ haloes, we also utilize additional six simulated stellar haloes obtained from J08, which we refer to as artificial haloes. These haloes are also simulated in the exact same way as the $\Lambda \mathrm{CDM}$ haloes except that these haloes have artificial accretion history, that is they are constructed by collaging accretion events from a library of satellites involved in creating the $\Lambda \mathrm{CDM}$ haloes described previously, but chosen to acquire the following properties:

(i) rad: a radial halo built from events predominantly on radial orbits, i.e. ratio of the angular momenta of the orbit to a circular orbit of same energy, $\epsilon<0.2$

(ii) circ: a circular halo built from events predominantly on circular orbits, i.e. ratio of the angular momenta of the orbit to a circular orbit of same energy, $\epsilon>0.7$

(iii) old/ancient: an old halo built from events entirely accreted, the time since accretion, more than $11 \mathrm{Gyr}$ ago,

(iv) young/recent: a young halo built from events entirely accreted, the time since accretion, less than 8 Gyr ago.

(v) highl: a high in luminosity halo built from events that were more luminous than $10^{7} \mathrm{~L}_{\odot}$, and

(vi) lowl: a low in luminosity halo built from events that were less luminous than $10^{7} \mathrm{~L}_{\odot}$.

These haloes possess roughly $10^{9} \mathrm{~L} \odot$ stellar tracers. The artificial haloes are not cosmologically motivated as they are constructed from a collage of different satellites that have been evolved in different host potential having different concentration parameter $c$. Hence, the intrinsic mass profiles of the resultant artificial haloes cannot be properly defined. Therefore, we only use these haloes to study the sensitivity of the properties of sub-structures in the halo to accretion history.

All of the above $\Lambda \mathrm{CDM}$ as well as artificial haloes are built up from the disruption of $>300$ satellites. Among these satellites, only the 15 largest satellites account for 75-90 per cent of the luminosity of the halo. As such, we are only interested in the tracer population dispersed from disrupted satellites that orbit and, hence, trace the underlying potential of the parent galaxy. Therefore, we exclude the particles that are still bound to the host satellites as they are not in equilibrium with the parent galaxy.

In Fig. 2, we show the radial velocity distributions of the stellar tracer population of the simulated stellar haloes as a function of 
$\Lambda$ CDM halos

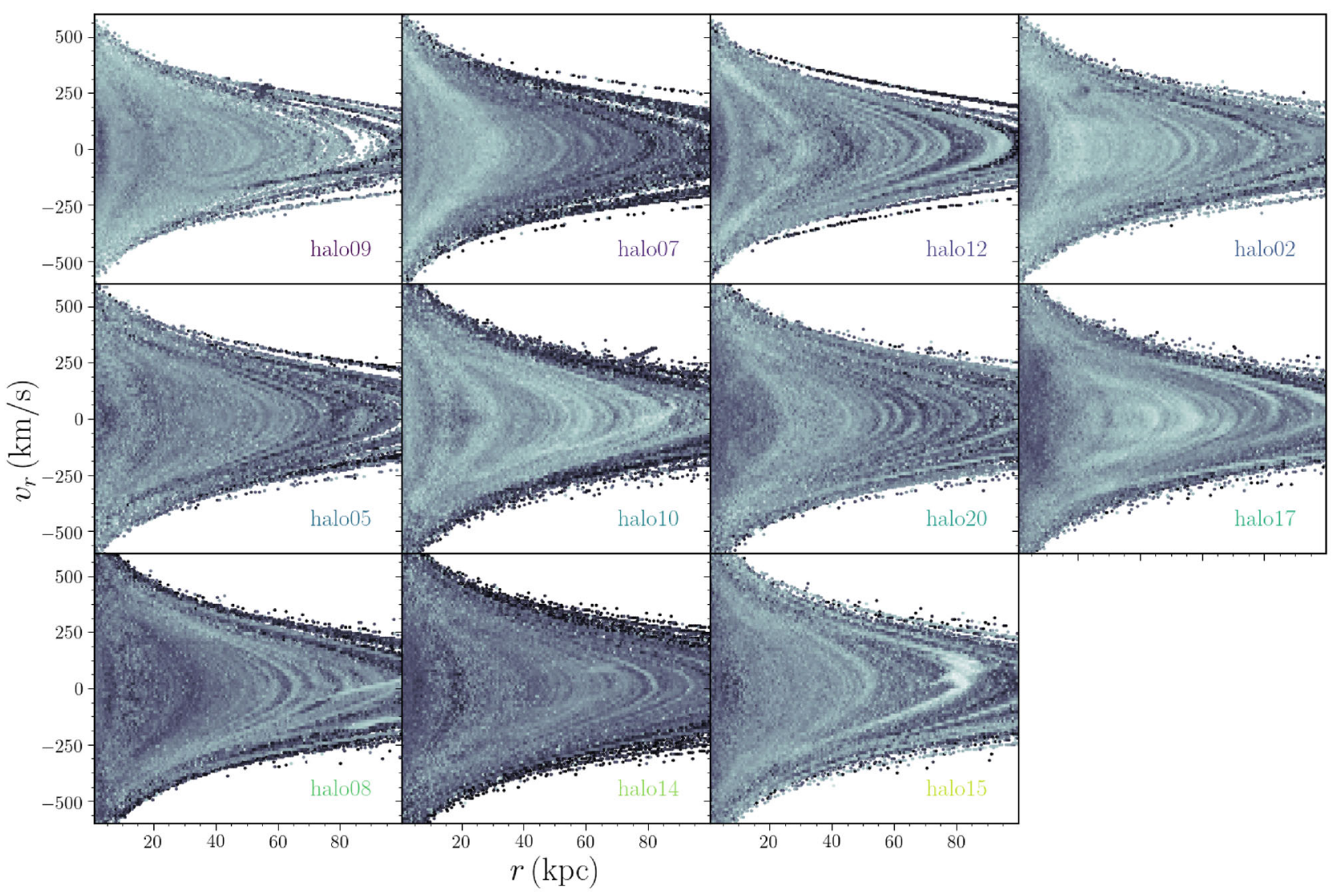

Artificial halos

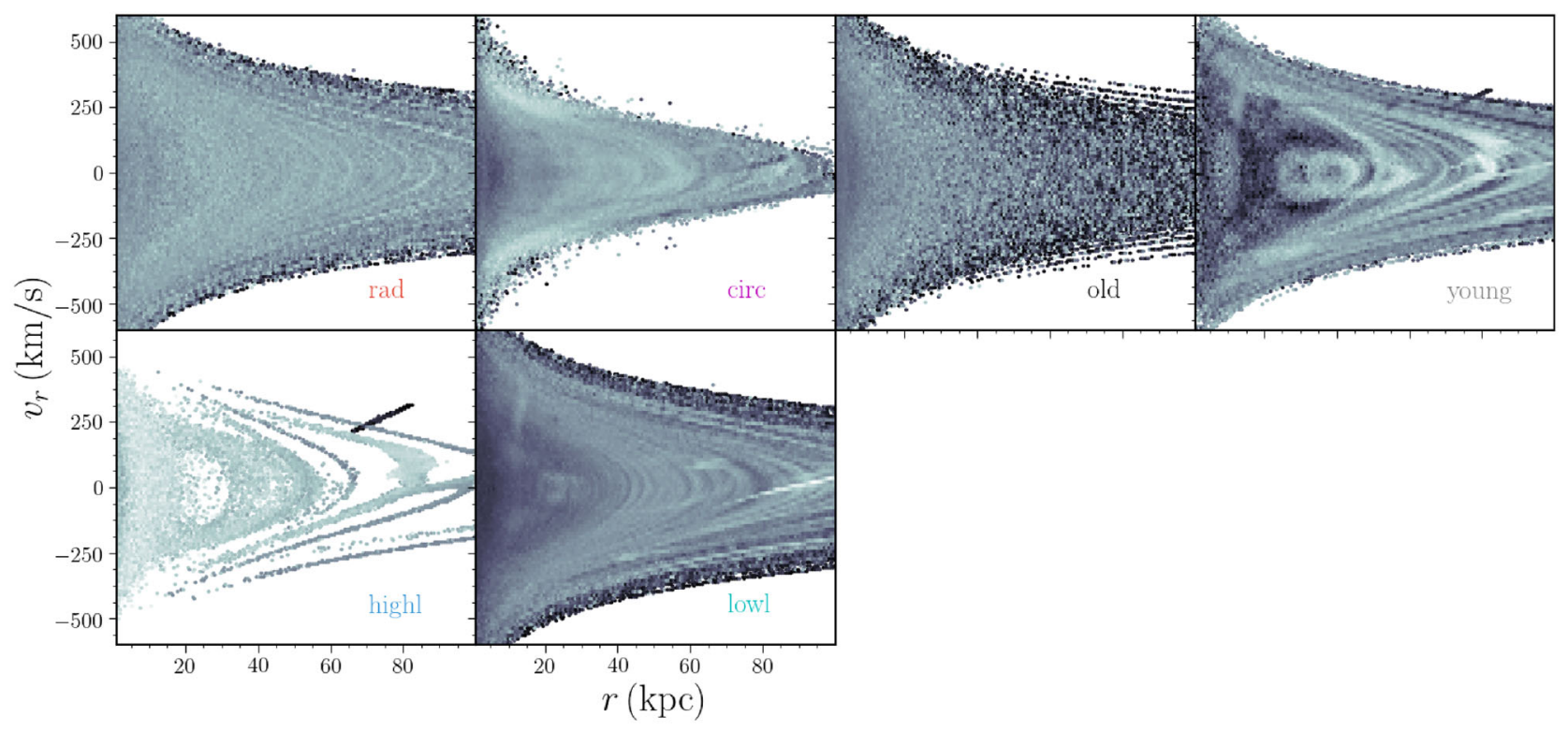

Figure 2. Radial phase-space diagrams ( $v_{r}$ versus halo-centric radius $r$ ) of all the simulated stellar haloes from the BJ05 (top panels) and J08 (bottom panels) simulations within $r / \mathrm{kpc} \in[1,100]$.

halo-centric distance $r / \mathrm{kpc} \in[1,100]$. In the bottom panel, where we show the artificial haloes, we see that the tracers of the young halo are relatively less relaxed compared to the $\mathrm{old}$, $\mathrm{rad}$, and $l o w l$ haloes, which are better phase-mixed and is as expected. The top panel of the figure where we show the $11 \Lambda \mathrm{CDM}$ haloes demonstrates diverse distribution of sub-structures, akin to the distinct accretion history of the haloes. The effective number of star particles in these simulated stellar haloes ranges between $2 \times 10^{4}$ and $8 \times 10^{4}$, exception to this are the rad, circ, and lowl haloes, which have $10^{5}$, $10^{5}$, and $6 \times 10^{5}$ particles, respectively. 


\section{DYNAMICAL MASS: JEANS FORMALISM}

\subsection{Formulary}

The Jeans (1915) equation for a pressure-supported, collision-less and spherically symmetric dynamical system in equilibrium is customarily expressed in spherical polar coordinates as follows:

$M_{\text {Jeans }}(<r)=-\frac{r \sigma_{r}^{2}}{G}\left(\frac{\mathrm{d} \ln \rho}{\mathrm{d} \ln r}+\frac{\mathrm{d} \ln \sigma_{r}^{2}}{\mathrm{~d} \ln r}+2 \beta\right)$

(Xue et al. 2008; Deason et al. 2012; Kafle et al. 2012; Richardson $\&$ Fairbairn 2013; King et al. 2015). When number density $\rho(r)$, radial velocity dispersion $\sigma_{r}(r)$, and velocity anisotropy $\beta(r)$ runs of the dynamical tracer populations are known, one can substitute them in the Jeans equation to derive the underlying mass $M_{\text {Jeans }}(<r)$ (or $M$ for conciseness) distribution of the system. Here, $r$ stands for a distance from the centre of the system, and the velocity anisotropy is expressed as

$\beta=1-\frac{\sigma_{\theta}^{2}+\sigma_{\phi}^{2}}{2 \sigma_{r}^{2}}$,

where $\sigma_{\theta}$ and $\sigma_{\phi}$ are angular and azimuthal velocity dispersions of the tracer populations. The $\beta$ parameter can have values between $[-\infty, 1]$, where $\beta<0, \beta=0$, and $\beta>0$ signify tangential, isotropic, and radial system, respectively.

We rearrange equation (1) and express it in terms of the radial pressure term $\left(p=\rho \sigma_{r}^{2}\right)$ as follow:

$M(<r)=-\frac{r}{G}\left[\sigma_{r}^{2}\left(\frac{\mathrm{d} \ln p}{\mathrm{~d} \ln r}+2\right)-\left(\sigma_{\theta}^{2}+\sigma_{\phi}^{2}\right)\right]$

Compared to equation (1), equation (2) has one less differentiation operation, and also, it does not contain the $\beta$ term explicitly, which makes the analysis simpler. We numerically compute the terms in the right-hand side of the equation in concentric radial shells. The density $\rho_{i}$ of the $i$ th bin between radii $r_{i}$ and $r_{i+1}$ is calculated directly from the frequency of the corresponding bin $n_{i}$ using

$\rho_{i}=\frac{n_{i}}{(4 \pi / 3)\left(r_{i+1}^{3}-r_{i}^{3}\right) \sum_{i} n_{i}}$.

Here, we have rescaled the density by the total number of tracers $\left(\sum_{i} n_{i}\right)$ and this is to facilitate comparison amongst the simulated haloes with varying number of tracers. The scaling has no effect in the final mass measurement as the Jeans equation only demands for the logarithmic slope of the density distribution and not its normalization. In our case, where star particles are essentially the accreted DM tracer particles with individual luminosity weight $\left(w_{i}\right)$, we replace the frequency $n_{i}$ with the effective sample size $=$ $\left(\sum_{i} w_{i}\right)^{2} / \sum_{i} w_{i}^{2}$. Similarly, in this case, we calculate the weighted velocity dispersions using

$\sigma_{\mathrm{w}}=\sqrt{\frac{\sum_{i} w_{i} v_{i}^{2}}{\sum_{i} w_{i}}-\left(\frac{\sum_{i} w_{i} v_{i}}{\sum_{i} w_{i}}\right)^{2}}$,

where, $v_{i}$ represents $v_{r} / v_{\theta} / v_{\phi}$, spherical polar component of the velocity vector of the $i$ th star particle.

Irrespective of the chosen form of the Jeans equation, there are some sources of noise, which are inevitable as they akin to physical processes. For example, the number of dynamical tracers drop sharply at large radii, resulting large scatter in $\rho$ and $p$ estimates. Additionally, dispersed (not yet fully phase-mixed) substructures are ubiquitous feature of the $\Lambda \mathrm{CDM}$ haloes that can be present at different radius. In such radial shells, velocity dispersions and density measurements are likely to be biased and dominated by the substructures. Consequently, this can bias the slope of the radial pressure $(\mathrm{d} \ln p / \mathrm{d} \ln r)$ as well. Also, in the very inner $r \lesssim 1 \mathrm{kpc}$ region of the simulated stellar halo, there is usually a sharp rise and then fall in the velocity dispersion profiles. The sudden change in the kinematics for a small change in radius makes the pressure-slope $(\mathrm{d} \ln p / \mathrm{d} \ln r)$ poorly measured. Moreover, in reality, this region is completely dominated by the bulge and the disc, where it is not relevant to consider finding the potential from the halo tracers. For these reasons, we restrict our study to $1<r / \mathrm{kpc}<100$.

Finally, we estimate the scatter around our all measurements using the bootstrap scheme, which is implemented by constructing 100 sub-samples (with replacement) of the data set. We consider the mean and standard deviation of the bootstrapped measurements at each bin as our best measurement and associated uncertainties of the relevant physical quantities, respectively. Moreover, to quantify the bias in the mass measurement, we define

mass offset(in per centage $)=100 \times \frac{M-M_{\text {True }}}{M_{\text {True }}}$,

where $M_{\text {True }}$ denotes the intrinsic mass profile of the parent galaxy (shown in Fig. 1), whereas $M$ represents the galaxy mass estimated from the Jeans formalism.

\subsection{Tests with a featureless simple model}

To investigate inherent biases in our Jeans formalism, first we test the scheme on toy data sampled from an ergodic Hernquist (1990) model with scalelength $a=15 \mathrm{kpc}$, total mass $=1.4 \times 10^{12} \mathrm{M}_{\odot}$, and isotropic velocity distribution $(\beta=0)$. By nature, the data is smooth, i.e. devoid of any sub-structures or tidal features, and therefore allows us to understand, if any, intrinsic bias in our scheme. To facilitate comparison to our main results with simulated stellar haloes, we generate a sample roughly of the same order of magnitude, i.e. $10^{5}$.

In our context where we are probing an order of magnitude range in distances and also where sample size sharply declines at large distance, the logarithmic binning in radius is more appropriate compared to a linear equal width or equal number binning schemes. Therefore, we divide our sample into 15 logarithmically spaced radial shells, and present the key spatio-kinematics properties of the tracers in Fig. 3. Intrinsically, all three velocity dispersion profiles $\left(\sigma_{r}, \sigma_{\theta}\right.$ and $\left.\sigma_{\phi}\right)$ of the tracer populations are identical; therefore, to make them visually distinct, we systematically shift the angular and azimuthal dispersions by $\pm 5 \mathrm{~km} \mathrm{~s}^{-1}$ (Fig. 3a). In the figure thickness of all, but except red-dashed, lines presented in panels (a)-(e) show the $1 \sigma$ uncertainty levels. Panel (e) compares the underlying mass distribution using the Jeans formalism with the intrinsic values of the system, and finally, panel (f) shows the residual in the mass measurement. In summary, the exercise demonstrates that the spatio-kinematic runs of the test data and the underlying mass distributions of the system at $r>5 \mathrm{kpc}$ can be recovered well enough with negligible mass offset of $0.14_{-1.9}^{+1.3}$ per cent, where average dispersion is consistent with the median random uncertainties of 1.6 per cent. However, due to abrupt change in the slopes of the velocity dispersions, the mass profiles in the inner region deteriorates. Additionally, we also achieve similar level of accuracy for alternative cases such as when the velocity distributions of the data are assumed to be radial ( $\beta=0.5$ and 0.9$)$ or tangential $(\beta=-0.5$ and -1.0 ). 

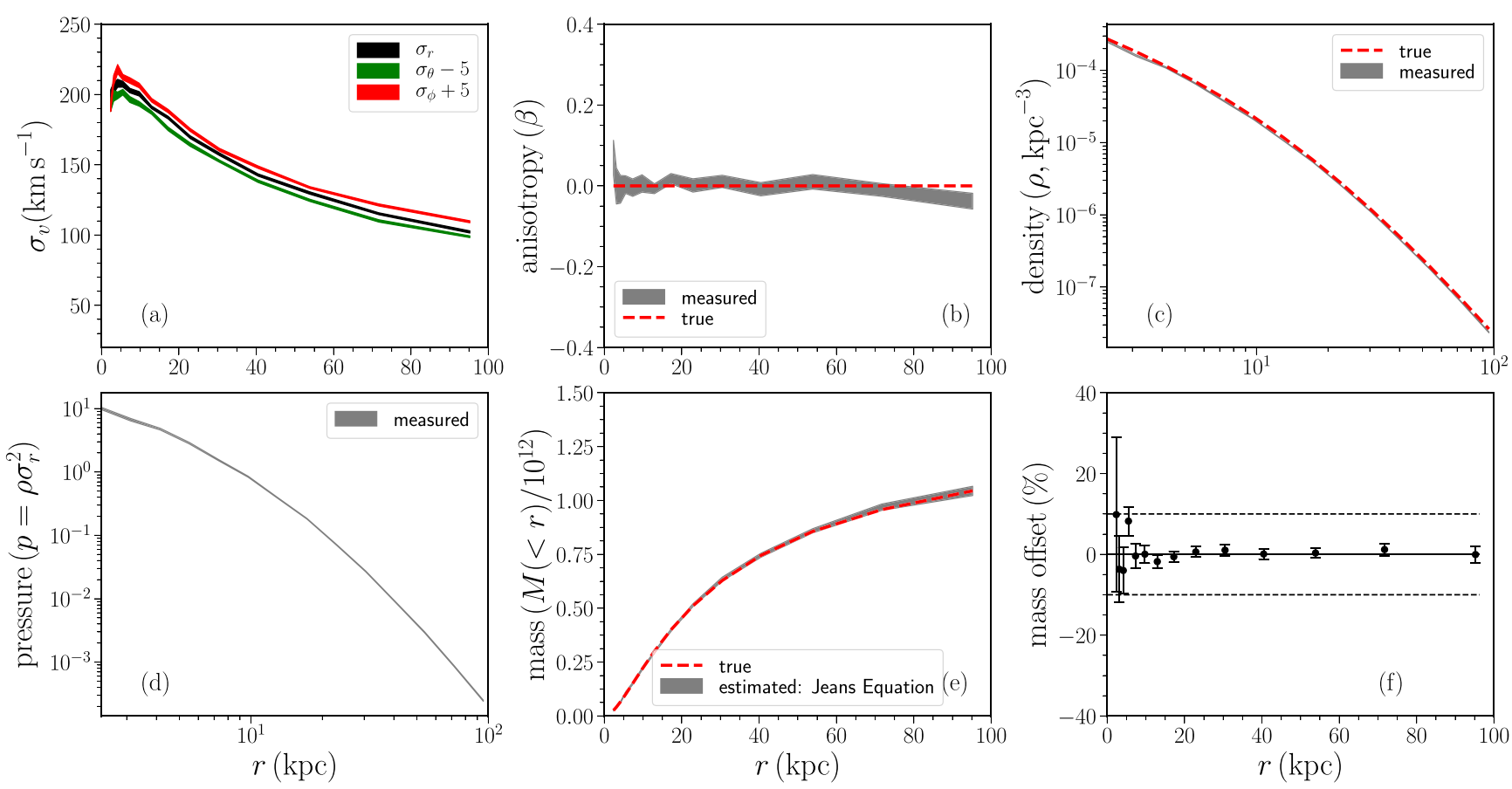

Figure 3. Jeans formalism implemented on a synthetic data sampled from the Hernquist distribution. Panels show (a) radial (red line), angular (green line), and azimuthal (black line) velocity dispersions; (b) velocity anisotropy (grey band: measured, red dashed-line: the input profile); (c) number density (grey band: measured, red dashed-line: the input profile); (d) measured radial pressure; (e) cumulative mass (grey band: reconstructed from the Jeans equation, red dashed-line: the input profile); and (f) percentage error in the mass measurement (black dots) with dashed lines at \pm 10 per cent as a guide.

\section{RESULTS}

\subsection{Spatio-kinematic profiles of the simulated stellar haloes}

In Fig. 4, we demonstrate the key spatial and kinematic properties of the tracer populations of the $11 \Lambda \mathrm{CDM}$ (top panels) and 6 artificial (bottom panels) haloes binned, respectively, in 25 concentric radial shells. As labelled in the figure, each solid line of different colour represents different halo. Panels (a) and (b) show the measured radial velocity dispersion $\sigma_{r}(r)$, panels (c) and (d) show the measured angular ${ }^{2}$ velocity dispersion $\sigma_{\theta}(r)$, and panels (e) and (f) show the corresponding velocity anisotropy $\beta(r)$ profiles of the tracer populations. Similarly, panels ( $g$ and $h$ ) and ( $i$ and $j$ ) show the measured number density and pressure distributions of the tracer populations. Finally, substituting the above measured tracer properties into the Jeans formalism (equation 2), we estimate the mass profiles of the parent galaxy, which are shown with solid lines in panels (k) and (l). For an easy comparison, in panels (k) and (l), we also overplot the inherent mass profiles of the parent galaxy, assuming maximum (red dashed-line), minimum (green dashed-line), and average (black dashed-line) values of concentration adopted from Fig. 1. The width of the lines in panels (a-j) depict the scatter around the relation obtained from bootstrapping, whereas to avoid further cluttering, we only show mean relations in panels (k) and (1). There are a few trends we note in Fig. 4. For example, both the $\sigma_{r}$ and $\sigma_{\theta}$ (also $\sigma_{\phi}$, not shown in the figure) attain highest value for small $r$ and vice-versa, which turn-over at $r \simeq 5 \mathrm{kpc}$. Also, all of the haloes except the circ have predominantly radial orbits i.e. $\beta>0$ (see panels e and f). Furthermore, it can be visually attested

\footnotetext{
${ }^{2}$ To save the space, we do not show the azimuthal velocity dispersion $\sigma_{\phi}$,
} which generally has runs identical to $\sigma_{\theta}$. that the logarithmic density distributions $\rho(r)$ of the tracer particles seem to follow a double power law with a break at $r \sim 20 \mathrm{kpc}$.

\subsection{Mass reconstruction from tracer populations}

For clarity, in Fig. 5, we recast the mass profiles of the parent galaxy of $11 \Lambda \mathrm{CDM}$ (top panels) and the 6 artificial (bottom panels) haloes, taken originally from panels (k) and (1) of Fig. 4. The magenta bands with solid lines show the mass profiles of the parent galaxy reconstructed from the Jeans equation using the spatio-kinematic profiles of the stellar tracer populations. The bands around the lines show the bootstrapped uncertainties around the mean measurements. Furthermore, the intrinsic mass distribution of the parent galaxy is shown with the black dashed-line. As mentioned earlier in Section 2, the intrinsic mass profiles of the artificial haloes are not known as they are constructed from a mixture of satellites that have been evolved in different host potential. Therefore, in the case of artificial haloes, we only use the black dashed-lines as a rough guide for the purpose of a qualitative assessment, which are derived assuming the concentration of the artificial haloes to be an average concentration of the satellites that make up these haloes. The spikes in the mass profiles are far more prominent than the uncertainties in the measured values; therefore, statistical noise as a potential cause for the spikes can be ruled out. We now investigate the cause of these spikes.

We expect that the mass distributions of the parent galaxy monotonically increase as a function of radius. However, this is not strictly the case for our estimated mass profiles, again magenta lines shown in Fig. 5. At many places, the mass profiles are bumpy (e.g. halo 09 at $r \sim 60 \mathrm{kpc}$, halo 10 at $r \sim 70 \mathrm{kpc}$ ), and in some cases, the cumulative mass dips (e.g. halo 08 at $r>60 \mathrm{kpc}$, highl halo at $r>50 \mathrm{kpc}$ ), which is unphysical. Similarly, the mass profiles of the highl halo at $r \gtrsim 60 \mathrm{kpc}$ and young halo at all radius are also 

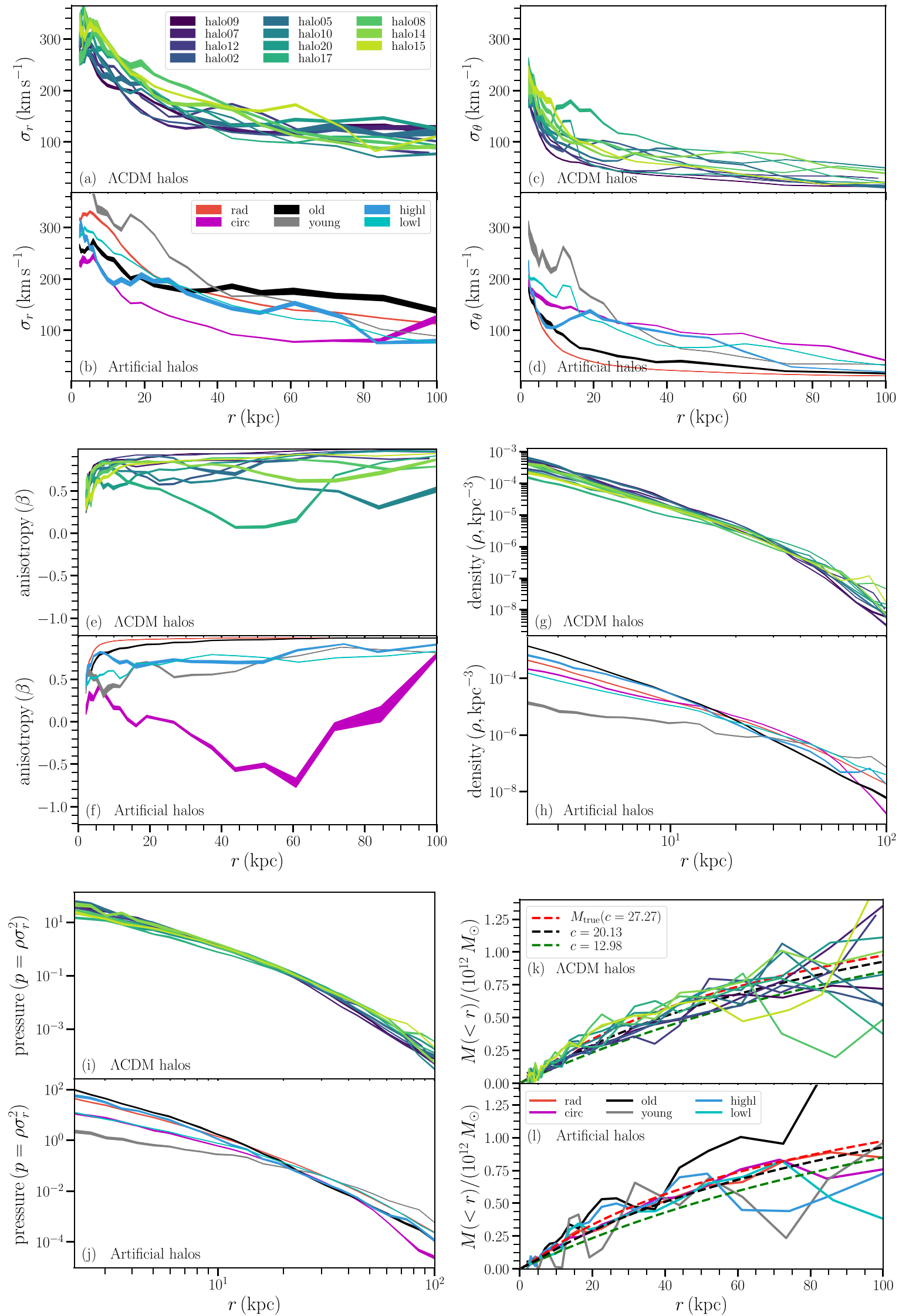

Figure 4. Key spatio-kinematic properties of the tracer populations of the simulated stellar haloes and inferred mass distribution of the parent galaxy taken from BJ05 and J08 simulations. 

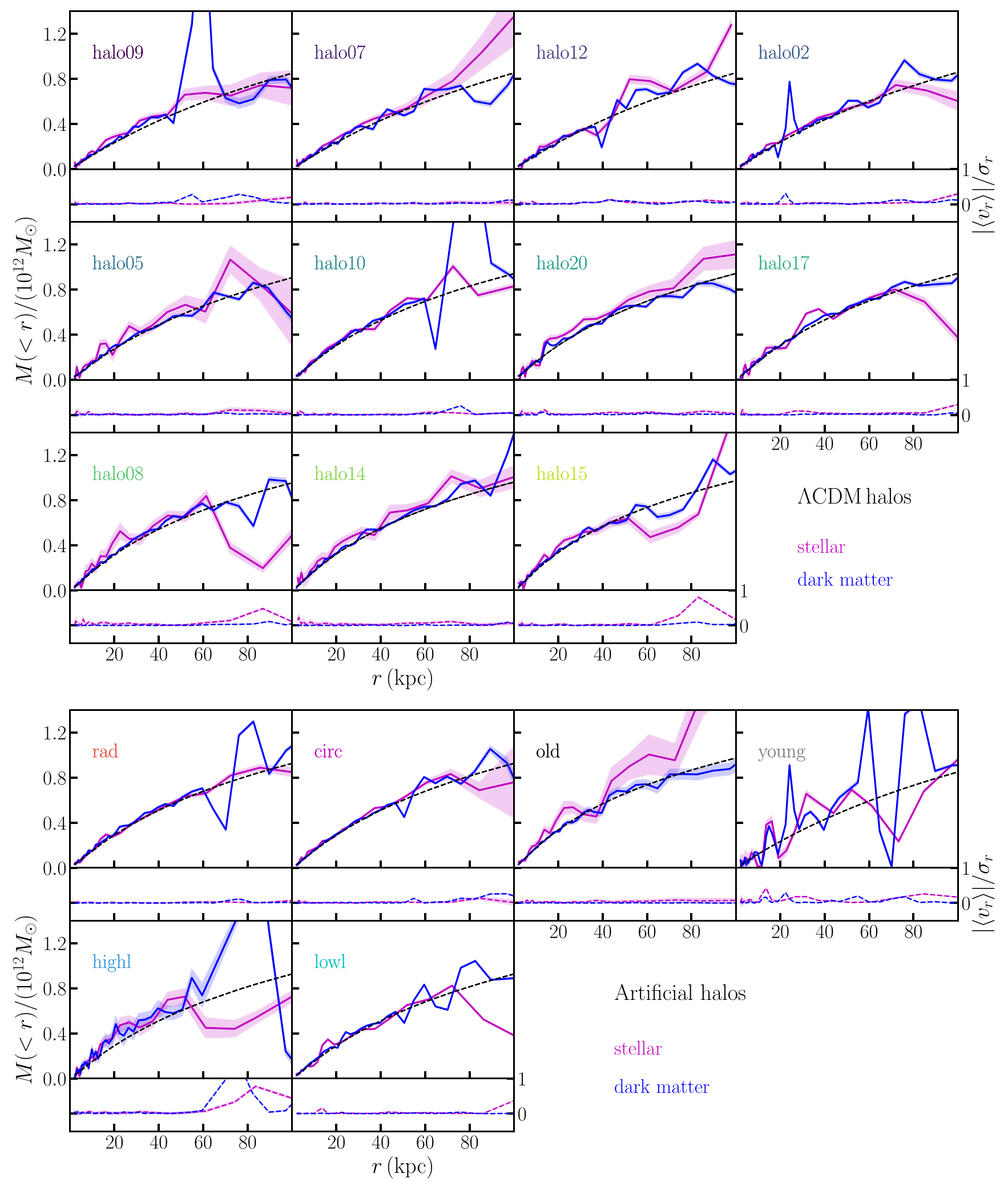

Figure 5. Mass profiles of the parent galaxy obtained from the Jeans formalism using the spatio-kinematic profiles of the tracer populations. Black dashed lines show the intrinsic mass profile of the parent galaxy, whereas magenta and blue solid lines are estimated masses of the galaxy when stellar and accreted DM particles are used as dynamical tracers, respectively. The magenta and blue dashed lines shown in small panels at ordinates $\simeq 0$ are respective measurements of $\left|\left\langle v_{r}\right\rangle\right| / \sigma_{r}$. The bands of corresponding colours around the blue and magenta lines show the associated uncertainties obtained from the bootstrapping. 
bumpy. This discrepant output of our scheme can be understood in conjunction with the radial phase-space diagram shown in Fig. 2. It is clear from this figure that at some intermittent radius, satellites are just dispersing (say, halo 15 at $r \sim 80 \mathrm{kpc}$, halo 09 at $r \sim 60 \mathrm{kpc}$ etc.). In such radial shells, we will measure biased $\sigma_{r}$ and $\rho(r)$, and hence, the Jeans equation locally fails here as it demands velocity dispersions of stars evenly populating the velocity range not one biased by clumps. The young halo (built from recent $<8 \mathrm{Gyr}$ ago events), which have not had enough time to fully relax, suffers the worst from this scenario. Also, highl halo (dominated by the massive accretion events) shows significant undulations in mass profiles at large radius. On the contrary, haloes such as halo14 does not contain any dominant sub-structures and, hence, result a wellbehaving mass profile. Similarly, the haloes such as old and lowl, which are expected to be better phase-mixed haloes by construction, have comparatively smoother mass profiles.

In the end, we like to validate if the erratic mass measurements at intermittent radii are due to the presence of sub-structures that are out of equilibrium. The mean radial velocity $v_{r}=0$ is a necessary condition for a system in equilibrium (although not a sufficient condition). So, $\left|\left\langle v_{r}\right\rangle\right| / \sigma_{r}$ can be used to gauge the departure from equilibrium. Note, for $N$ stars in a bin, $\left|\left\langle v_{r}\right\rangle\right| /\left(\sigma_{r} / \sqrt{N}\right)$ is a better measure of the statistically significance of departure of $v_{r}$ from zero, as it takes the effect of Poisson noise into account. However, we choose to study $\left|\left\langle v_{r}\right\rangle\right| / \sigma_{r}$ as it is physically more meaningful and significant. From equation (1), we get

$\delta M / M \simeq 2 \delta \sigma_{r} / \sigma_{r} \simeq 2 v_{r} / \sigma_{r}$

assuming that the change in dispersion $\delta \sigma_{r}$ is of the order of $v_{r}$. Hence, $v_{r} / \sigma_{r}$ is useful to gauge if a change in $\left|\left\langle v_{r}\right\rangle\right| / \sigma_{r}$ is enough to explain a corresponding change in mass profile. The magenta dashed lines in all the tiny panels of Fig. 5 show the $\left|\left\langle v_{r}\right\rangle\right| / \sigma_{r}$ profile. Additionally, to check that a spike in $\left|\left\langle v_{r}\right\rangle\right| / \sigma_{r}$ is statistically significant and not caused by Poisson noise, we show the dispersion with band around $\left|\left\langle v_{r}\right\rangle\right| / \sigma_{r}$ profile using bootstrapping. We find that the erratic spikes seen in the mass profiles at many places, e.g. halo09 at $\sim 55 \mathrm{kpc}$, halo02 at $\sim 20 \mathrm{kpc}$, coincide with spikes in $\left|\left\langle v_{r}\right\rangle\right| / \sigma_{r}$ runs. Moreover, we see that the mass reconstructions outside $r>60 \mathrm{kpc}$ generally deteriorate for all the haloes, which is mainly because of the paucity of mass tracers and dominance of unrelaxed sub-structures in the outskirts.

In the figure, the blue solid and dashed lines are the corresponding reconstructed mass profiles of the parent galaxy when the accreted DM particles are used as a dynamical tracer. The dark matter particles are not directly observable and the discussion of this case is not useful for pragmatic reasons. However, for the completeness reason, we briefly present and discuss the spatio-kinematic profiles and error analysis of this case in the Appendix 6. For almost all the haloes, we observe that the stellar tracers show comparatively less ridges in the reconstructed mass profiles compared to the cases when DM tracers are used. This is because the DM tracer populations have large number of prominent sub-structures with zero luminosity, and hence zero stellar mass.

The intermittent noise in the inferred mass distributions can be reduced by fitting a smooth parametric models to the velocity dispersions and pressure/density runs that enter the Jeans analysis. We restrain from doing so mainly because a smooth model cannot capture the impact of sub-structures in the mass profiles. Also, it leads to a natural question of what are the good models for the dispersion profiles, number density etc. Therefore, we decided to use the binned data directly.
A takeaway point from Fig. 5 is that in overall the shape of the mass profiles of the parent galaxy of the simulated stellar haloes (except for the flagged haloes such as highl, young), although bumpier at places where sub-structures locally dominate, can be recovered well using the Jeans analysis. Below we provide a more detail account of the biases in our overall mass reconstruction.

In Fig. 6, we present the error analysis of the mass measurements of the parent galaxy using the stellar tracer populations for all $11 \Lambda \mathrm{CDM}$ haloes. Panel (a) shows the mass offsets (defined in equation 5) in the estimated mass $M_{\text {Jeans }}$ compared to the intrinsic masses as a function of $r$. Similarly, in panel (b), we show the fractional uncertainty $\left(\sigma_{M} / M\right)$ on the estimated mass measured using bootstrapping as a function of $r$. Different colours of the circles or lines in panels (a) and (b) represent different haloes, the labellings consistent with Fig. 1. The average of the coloured lines in panel (b) is shown with the black dashed-line, whereas the black solid line is the dispersion profile of the mass offsets obtained from panel (a).

The mass offset is due to two sources: (a) random uncertainty in the estimator, which is mainly due to Poisson noise, and (b) the tracers not being in dynamical equilibrium with the potential in which they are orbiting. We can estimate the random uncertainty by bootstrapping and this is given by $\sigma_{M}$. We label the dispersion due to non-equilibrium effects by $\sigma_{\text {noneq. }}$. The dispersion in mass offset can then be written as

$$
\left(\frac{\sigma_{\text {mass offset }}}{M}\right)^{2}=\left(\frac{\sigma_{M}}{M}\right)^{2}+\left(\frac{\sigma_{\text {noneq }}}{M}\right)^{2} .
$$

If we consider the standard error of mean around the average $\sigma_{\text {mass offset }} / M$ relation in Fig. $6(\mathrm{~b})$, the solid black line coincides within the $2 \sigma$ confidence interval of the mean relation in the inner $r<10 \mathrm{kpc}$. The two relations tracing each other in this regime means that the dispersion in mass offset is dominated by random uncertainty due to Poisson noise and can be reduced by increasing the sample size of the tracers. Moreover, the large uncertainty in the inner $r<10 \mathrm{kpc}$ is of the least concern to us as we are mainly interested in measuring mass for $r \gtrsim 10 \mathrm{kpc}$. However, for $r \gtrsim 10 \mathrm{kpc}$, the dispersion in mass offset is consistently larger than the random uncertainty and the difference keeps on increasing with increase of $r$. This means that dispersion is dominated by non-equilibrium effects instead of random uncertainty, and this dispersion cannot be reduced by increasing the sample size or the precision of observables.

Finally, to quantify the bias and dispersion in the mass measurement, we present the distributions of the mass offsets in panel (c), where blue, orange, and black histograms show the cases with $r \geq$ $10 \mathrm{kpc}, r<10 \mathrm{kpc}$, and $r / \mathrm{kpc} \in[1,100]$, respectively. The median and the 16th and 84th percentile ranges are shown in the panels (denoted by per.). In essence, from panel (c), we conclude that in reconstructing the mass profile of the parent galaxy using the stellar halo tracer populations within $r / \mathrm{kpc} \in[10,100]$, the bias is $\sim 10$ per cent and the dispersion is $\sim 14$ per cent. When we include the results from the inner $r<10 \mathrm{kpc}$ as well, the bias remains the same whereas dispersion marginally increases to $\sim 20$ per cent. Additionally, we also confirm that the bias in the mass measurements is not introduced due to the consideration of mean motion while calculating the velocity dispersion in equation (4). Importantly from Fig. 6(b), we measure that the random error in mass measurement in the case of $r \gtrsim 10 \mathrm{kpc}$ is $\sim 7$ per cent that is approximately half of the dispersion ( $\sim 14$ per cent). Substituting this value in equation (7), we measure the contribution of non-equilibrium effect to be 12 per cent. To investigate this, we repeat our analysis with root-mean square velocity as a measure for the velocity dispersion, and we observe 

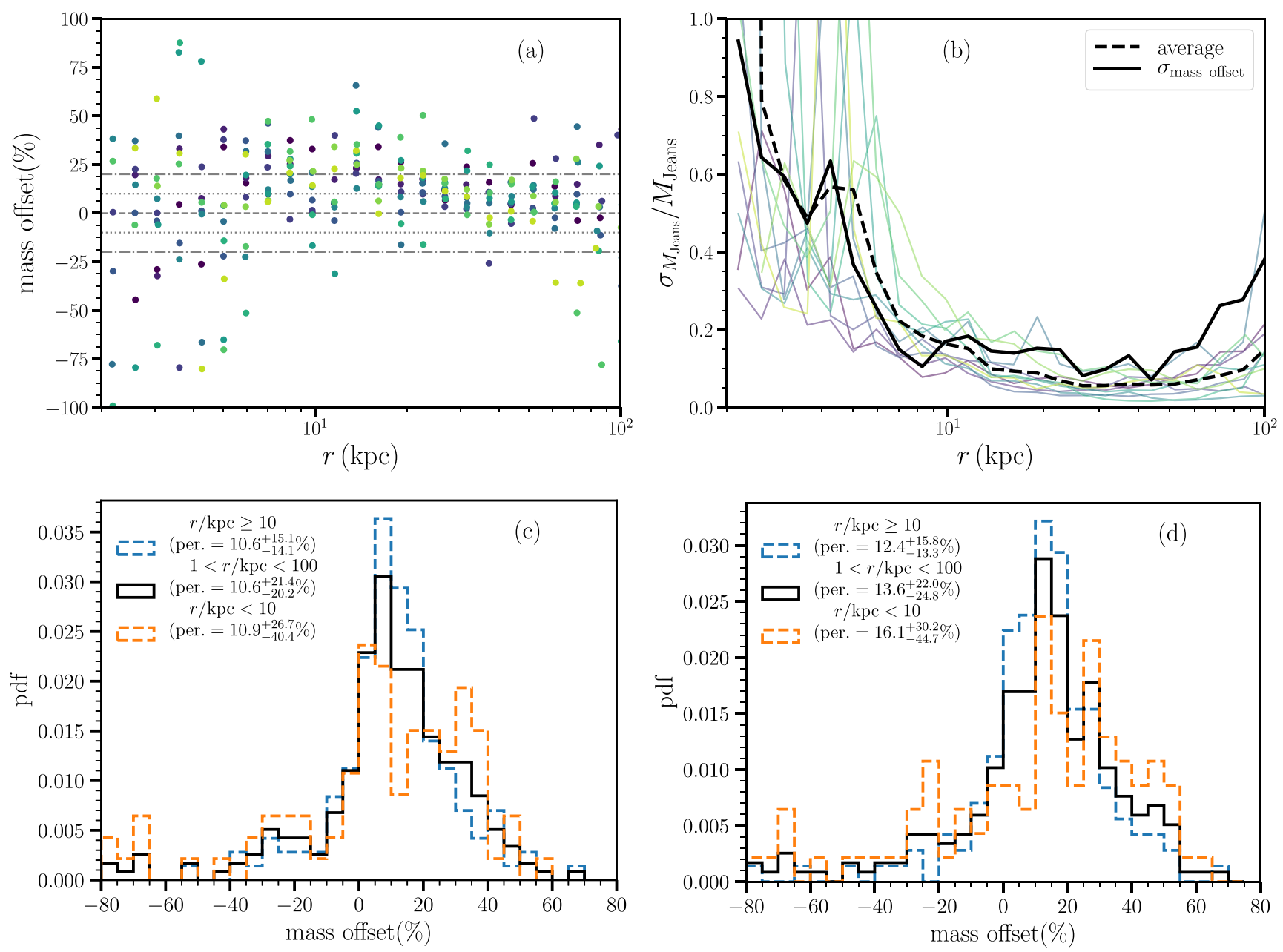

Figure 6. Error analysis of the estimated masses of the parent galaxy using the stellar tracer populations of the $11 \Lambda \mathrm{CDM}$ haloes. Panel (a) shows the percentage mass offset and panel (b) shows the random uncertainties in mass measurements, both as a function of distance, where different colours represent different haloes as labelled in Fig. 1. Panels (c) and (d) show distributions of the mass offset for cases where we, respectively, use standard deviation and root-mean square velocity as a measure for the velocity dispersion.

effectively similar bias in mass measurements as demonstrated in panel (d) of the figure.

\subsection{Deviation from spherical symmetry}

A generic prediction of structure formation under the $\Lambda \mathrm{CDM}$ paradigm is that galactic DM haloes are triaxial (e.g. Jing \& Suto 2002; Bailin \& Steinmetz 2005; Allgood et al. 2006; Vera-Ciro et al. 2014), which has been shown to become comparatively more spherical due to influence of the baryonic processes (Kazantzidis et al. 2004; Gustafsson, Fairbairn \& Sommer-Larsen 2006; Kazantzidis, Abadi \& Navarro 2010). The observations of the Galaxy show varying results. In this, study of debris of the Sagittarius dwarf tidal stream distributed on a great circle (Ibata et al. 2001) and also the bifurcation in the stream (Fellhauer et al. 2006) suggest near spherical Galactic DM halo. In contrast, the line-of-sight velocities of the stream favour a triaxial Galactic halo (Helmi 2004). More recently, Law, Majewski \& Johnston (2009) and Deg \& Widrow (2013), while fitting spatio-kinematics of the Sagittarius stream, find Galactic potential consistent to be triaxial and determine halo intermediate/major $(b / a)$ axis ratio of 0.83 and minor/major $(c / a)$ axis ratio of 0.67 . The constraints on the shape of the MW DM halo is still an unresolved subject; for additional constraints and further discussion of the topic, see the review by Read (2014).

As discussed earlier, the DM halo potential of the parent galaxy in BJ05 simulations is spherical. Therefore, to investigate the applicability of the spherical Jeans equation in the case of triaxial haloes, here we utilize the SURFS simulation (Elahi et al. 2017). The SURFS simulation is a suite of cosmological $N$-body simulations and we focus on its subset with box sizes $40 h^{-1} \mathrm{Mpc}$. The halo catalogues are constructed with the VELOCIraptor phase-space halo finder (Elahi, Thacker \& Widrow 2011). From the suRfs halo catalogue, we focus on low mass groups with virial masses ${ }^{3}$ of $\sim 10^{12.5} \mathrm{M}_{\odot}$, which provides us with a sufficient statistical sample of well resolved haloes composed of $\sim 5 \times 10^{4} \mathrm{DM}$ particles. Unfortunately, the SURFS simulation does not readily provide stellar tracer populations and therefore hindering a more realistic test. As such, it is not crucial at this point as we are only interested in understanding the sensitivity of the spherical Jeans equations in reconstructing the mass profiles of triaxial systems. We note that the mass range of

\footnotetext{
${ }^{3}$ Mass enclosed within the radius where the over density is 200 times the critical density of the universe.
} 


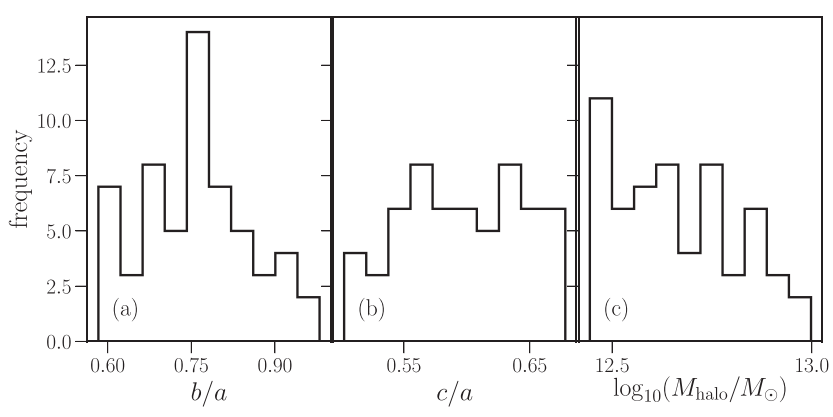

Figure 7. Distributions of (a) intermediate/major axis ratio (b) minor/major axis ratio, and (c) halo mass in logarithmic scale for triaxial DM haloes taken from the SURFS simulation.

the selected haloes we assume is slightly larger than a typical observational estimate of $\sim 10^{12} \mathrm{M}_{\odot}$ for the virial mass of the Galaxy (e.g. McMillan 2011; Kafle et al. 2014; Piffl et al. 2014; BlandHawthorn \& Gerhard 2016), a result of requiring haloes resolved with several tens of thousands of particles and the mass resolution of the simulation used. This mass difference between our mock haloes and the MW is irrelevant as in this narrow mass interval we expect that the number density and kinematic profiles of the haloes tracer populations remain self-similar. Critically, here we are only testing the applicability of the spherical Jeans formalism in mass reconstruction of the non-spherical haloes, therefore all we need is a set of realistic triaxial haloes.

Haloes within this mass range span a range of triaxiality parameters, ${ }^{4}$ from $b / a=[0.35,0.95]$ with typical values of 0.78 and with $c / a=0.7 b / c$ (Elahi et al. 2017, see fig.7). In the end, we only use haloes that have the triaxiality parameters reasonably close to the observed values of $b / a \approx 0.8$ and $c / a \approx 0.7$ for the MW DM halo (Law et al. 2009; Deg \& Widrow 2013). The distributions of the axis ratio and halo mass of our final sample of 58 haloes are shown in the top panels of the Fig. 7. Finally, we split the DM tracer populations of these haloes into 15 concentric spherical radial shells within $r \leq 100 \mathrm{kpc}$, and apply the spherical Jeans formalism (Section 3). The grey bands in Fig. 8(a) and (b) show the measured anisotropy and number density of the tracer populations, whereas panel (c) shows the derived cumulative mass profile of the underlying DM halo with the total mass of $\log _{10}\left(M_{\text {halo }} / \mathrm{M}_{\odot}\right)=12.9$ and axial ratios of $b / a=0.8$ and $c / a=0.7$. In panel (c), the overplotted red dashed-line represents a spherically averaged intrinsic mass profile of the halo. We see that the spherical Jeans formalism reconstructs the mass profile of the halo reasonably well, and results of the remaining 57 haloes are also consistent with this.

In Fig. 9, we provide an error analysis of the estimated masses of the triaxial haloes. The panel (b) demonstrates the mass offset (in percentage) for all the 58 haloes as a function of scaled radius $r / r_{\max }$, where $r_{\max }$ corresponds to the radius $r$ at which the circular velocity of the halo is maximum. Dashed lines in the panel highlight the offset of 10 per cent and 20 percent. The blue and orange histograms shown in panel (c) however show the distributions of mass offset at all scaled radii $r / r_{\max }>0.2$ and $>0.0$, respectively. The median mass offset at $r / r_{\max }>0$ is $-5.9_{-31.9}^{+16.4}$ percent, and $-2.4_{-9.9}^{+9.4}$ per cent at $r / r_{\max }>0.2$. The dispersion in the mass offset in the case of DM tracer populations of the $11 \Lambda \mathrm{CDM}$ haloes of

\footnotetext{
${ }^{4}$ the shape is calculated using the reduced inertia tensor (Dubinski \& Carlberg 1991; Allgood et al. 2006).
}

BJ05 at outer region ( $r \geq 10 \mathrm{kpc}$ ) is of similar level $\sim 7$ percent although the bias is positive here ( $\sim 3$ per cent). The dispersion obtained for $r / r_{\max }>0.2$ case is 8.7 per cent after adjusting for random uncertainty. In agreement with the BJ05 $\Lambda$ CDM haloes, here again we confirm that the biases in the mass measurements are not due to subtracting mean motion while calculating the velocity dispersion (in Equation 4). In the figure, we observe that generally in the inner $r / r_{\max }<0.2$ region, the scatter in the residual of the mass estimate is large. To further investigate the anomaly, in panels (d), (e), and (f), we show the relation of average mass offset at $r / r_{\max }<0.2$ as a function of $b / a, c / a$, and halo mass, respectively, and also provide their respective correlation measurements. We fail to find any correlations between the observed mass offsets and the aforementioned intrinsic properties of the haloes. Finally, from panel (a), where the ratios of dispersion to the estimated mass $M_{\text {Jeans }}$ of the triaxial haloes are shown, and similar to the earlier cases of BJ05 $\Lambda$ CDM haloes, here also we find that the large scatter in the residual of the mass estimate in the inner region is mainly due to Poisson noise.

In summary, we conclude that in the outer $r / r_{\max }>0.2$, the underlying mass profiles of the SURFs haloes can be determined with a bias of $\sim-2.4$ percent and a dispersion of $\sim 10$ percent. Note this dispersion includes the effects of triaxiality as well as departures from equilibrium.

\section{DISCUSSION AND CONCLUSION}

In this paper, we utilize the $11 \mathrm{MW}$ stellar haloes simulated in accordance with $\Lambda \mathrm{CDM}$ by BJ05, 6 additional simulated stellar haloes from $\mathrm{J} 08$ built to have artificial accretion histories dominated by events that are predominantly recent/old, on radial/circular orbits or having larger/smaller satellite mass, and 58 triaxial DM haloes obtained from the SURFS simulation (Elahi et al. 2017) to test the efficacy of the spherical Jeans (1915) formalism in predicting the mass distribution of the MW analogues in a $\Lambda \mathrm{CDM}$ universe.

In overall, using the spatio-kinematic profiles of the stellar tracer populations and the spherical Jeans equation, we recover the underlying mass distribution of the MW analogues in a $\Lambda \mathrm{CDM}$ universe within $r / \mathrm{kpc} \in[10,100]$, with a bias of $\sim 12$ per cent and a dispersion of $\sim 14$ per cent (12 per cent when adjusted for random uncertainty). Additionally, analysing triaxial DM haloes obtained from the SURFS simulation with intermediate/major axis ratio in range $[0.5,1]$ and minor/major axis ratio in range $[0.5,0.7]$, we are able to recover the underlying mass distribution of the haloes with a bias of $\sim-2.4$ per cent and a dispersion of $\sim 10$ per cent $(8.7$ per cent when adjusted for random uncertainty), in the outer $r / r_{\max }>0.2$ region of the haloes. Similar level of dispersion $(\sim 7$ percent or $\sim 6$ per cent when adjusted for random uncertainty) is also observed for the case of the DM haloes of BJ05 but with positive bias of $\sim 3$ per cent.

In perfect conditions, a spherical system in equilibrium, we can correctly reconstruct the mass profile and there is no bias and dispersion that can be accounted by random uncertainty on the estimated mass. When applied to data from simulations, we do see some bias and dispersion, meaning the bias and dispersion can be either due to system being aspherical and out-of-equilibrium or due to an unknown effect in simulations. We find little correlation of mass offsets with asphericity, so out-of-equilibrium effects seem to be the main cause behind the observed mass offsets. The out-of-equilibrium effects will shift the mass estimates at any given radius and this will lead to a non-zero dispersion. So the dispersion, in principle, sets a limit on the accuracy with which we can expect to measure mass at a given radius for any halo using the spherical Jeans equation. 

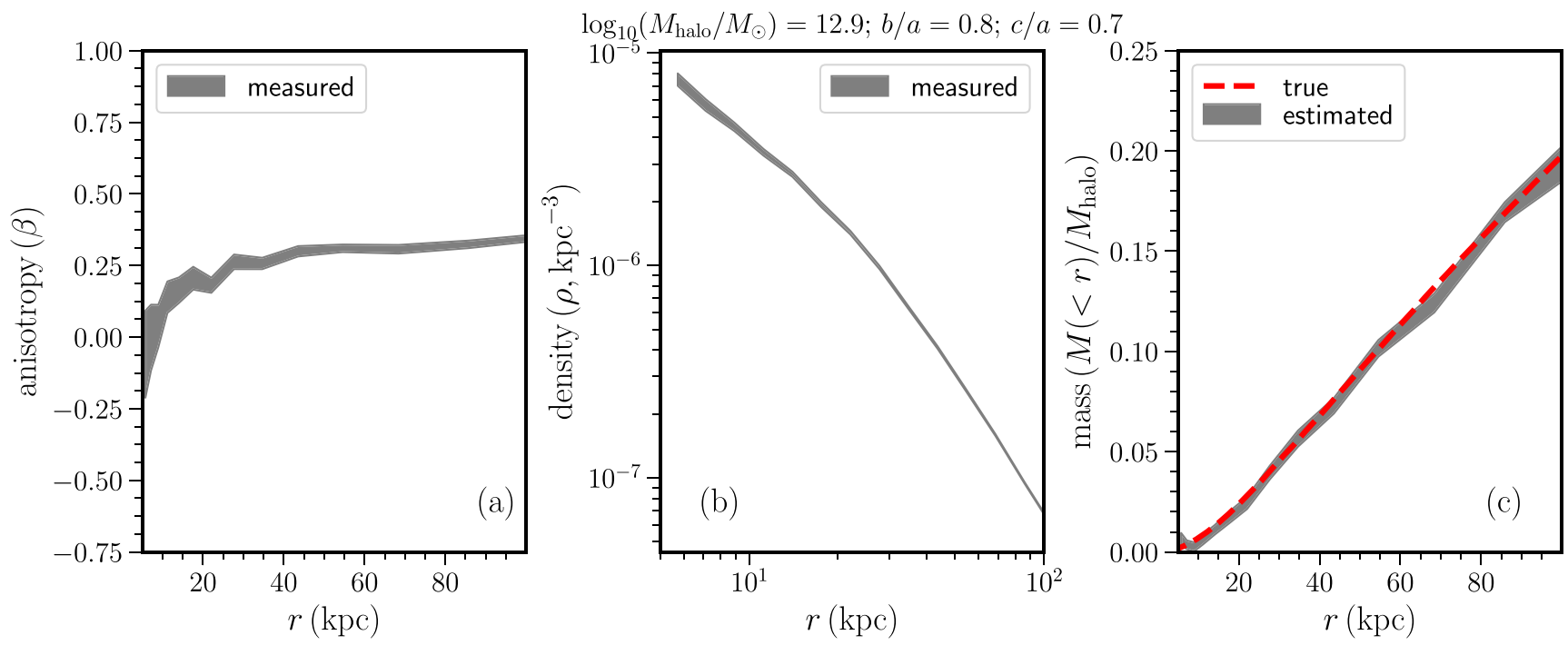

Figure 8. Spherical Jeans formalism in a triaxial DM halo from the suRFs simulation with velocity anisotropy, number density, and cumulative mass profiles of the halo shown in panels a, b, and c, respectively. The grey bands show the measured quantities with bootstrapped uncertainties, whereas red-dashed line in panel (c) represents the intrinsic mass distribution of the halo.
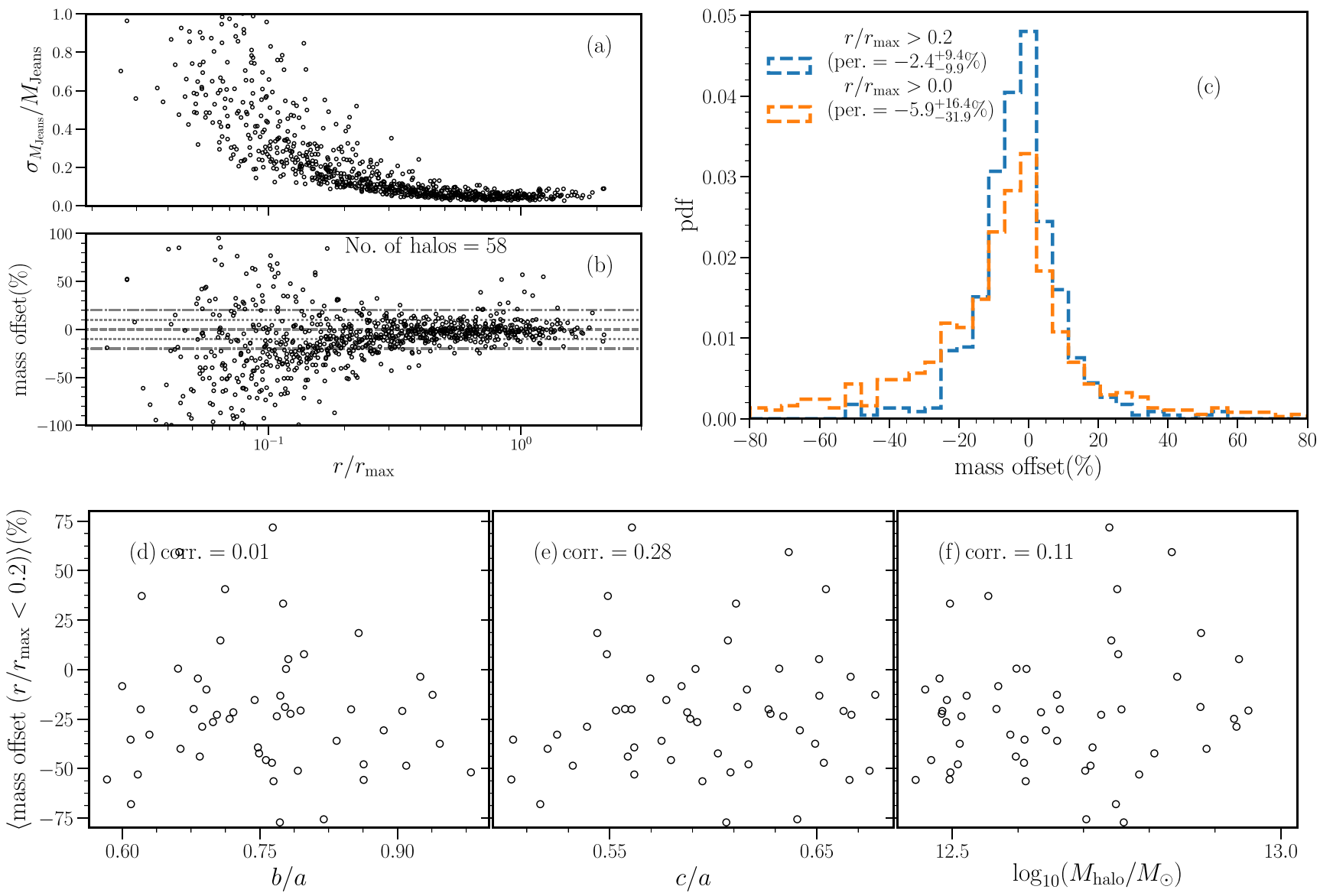

Figure 9. Error analysis of the mass measurement of triaxial DM haloes taken from the suRFs simulation. Panel (a) shows the random uncertainties in mass measurements and panel (b) shows the percentage mass offset both as a function of scaled radius for all 58 haloes, whereas panel (c) shows the distribution of the mass offset. Panels d-f show the correlation of the mass offset with triaxiality parameters and the mass of the halo. 
This limit is set due to the inherent nature of the $\Lambda \mathrm{CDM}$ haloes and is independent of the quality and the quantity of the observational data.

Using GALAXIA (Sharma et al. 2011b), a stellar population synthesis software utilizing Padova isochrones (Marigo et al. 2008), we estimate that, till the magnitude limit of 17 in $V$ band, Gaia will have more than 5 times the number of tracers that we have investigated here. So, the error on the mass estimates of the Galaxy using stellar tracers provided by the Gaia using the Jeans analysis will be limited by the non-equilibrium effects as well as uncertainties in observed distances and tangential velocities rather than the sample size.

The fact that the two different simulations (with DM particles as tracers) give similar level of dispersion (10 per cent for SURFS and 7 per cent for BJ05 haloes) in estimated masses is reassuring for our estimate of the dispersion. When applied to BJ05 stellar haloes (stellar particles as tracers), we get a higher dispersion (14 per cent). This is the case that is of practical use, as we observe stars rather than dark matter particles. The higher dispersion here is also as expected, since the stellar haloes have more sub-structures than dark matter haloes. Dispersion could also be due to random uncertainty associated with our mass estimator (effect of Poisson noise). We have shown that the random uncertainty is a factor of 2 smaller than the measured dispersion for all analysed cases. The total variance being the sum of squares of random and intrinsic scatter, the random uncertainty should make very little contribution to the dispersion. Taking the random uncertainty into account, we estimate the intrinsic scatter due to non-equilibrium effects to be 12 percent for the case of stellar haloes.

The interpretation of bias is less obvious. Naively, we expect the bias to be zero, at a given radius, the non-equilibrium effect can shift the mass in either direction (as the quantities on the right-hand side of equation (1) can shift in either direction). For the SURFS as well as BJ05 dark matter haloes, although not negligible but the bias is a factor of two smaller than the dispersion. For the BJ05 stellar haloes, the bias is much higher but still less than the dispersion. The sign of the bias is different for the SURFS and BJ05 simulations, and we have not been able to find a reason for it. However, the bias is significant only if we treat the measurement at each radius as independent and this might not be true. Typically, a few luminous/massive accretion events dominate in shaping the overall properties of a given the stellar halo. Specifically, the fraction of material in sub-structures is dominated by a few luminous accretion events (Sharma et al. 2011a, section 4.4). So mass measurements of a given halo at different radius can be correlated. Given that we only have 58 independent haloes for the SURFS and 11 for BJ05, the measured biases are less than twice of (disperion $/ \sqrt{\text { number of haloes) }}$ and are within the $2 \sigma$ limit. For the case of stellar haloes, the bias is higher but still within $2.5 \sigma$ limit. We note that in BJ05 simulations, a stellar halo is created by assigning unequal weights to dark matter particles. Although less likely (as after getting disrupted the particles of a satellite behave more or less independently) but it is worth exploring in future if this weighting scheme can introduce a systematic bias. Another possibility could be that the bias is a reflection of the initial conditions used to generate the accretion history of the halo. The accreting satellites tend to be closer to (or further from) peri- or apo-centre than one would expect for a phase-mixed population, meaning that their radial velocities are lower (or higher) than in that case. When the satellites disrupt and get fully phase-mixed, the system will be in equilibrium and the bias would vanish. However, here we are analysing a partially relaxed system, as evidenced by the presence of significant amount of sub-structure, and this can lead to a non-zero bias. For example, if we consider the simple spherical collapse model of the formation of a dark matter halo, at turn around the total energy is mostly potential, whereas at the collapse, it is kinetic. Applying Jeans equation here will give systematically lower mass at the turn around stage and higher mass at the collapse stage. The fact that the initial conditions are different in SURFS and BJ05 simulations could possibly lead to different biases in them.

Additionally, the investigation of the simulated stellar haloes with artificial accretion history suggests that the young halo built from events less than $8 \mathrm{Gyr}$ and the halo dominated by high luminosity $\left(<10^{7} \mathrm{~L} \odot\right)$ accretion events show the most undulations in the mass profiles and, hence, are the most error prone cases to apply the Jeans analysis. This is due to significant amount of unrelaxed sub-structures inherently present in these haloes. On the contrary, a halo dominated by less luminous $\left(<10^{7} \mathrm{~L} \odot\right)$ accretion events, awell-mixed halo, provides good scenario to apply the Jeans formalism.

\section{ACKNOWLEDGEMENTS}

PRK is funded through Australian Research Council (ARC) grant DP140100395 and the University of Western Australia Research Collaboration Awards PG12104401 and PG12105203. We like to thank the referee for constructive and insightful comments that helped to improve the paper significantly. We also thank Prof. Chris Power and Dr Claudia Lagos for providing the surfs data, and Prof. Geraint Lewis and Dr Luke Davies for discussions related to the paper. Software credit: IPYTHON (Pérez \& Granger 2007), MATPLOTLIB (Hunter 2007), SEABORN (Waskom et al. 2014), PANDAS (McKinney 2012), NUMPY (van der Walt, Colbert \& Varoquaux 2011), and SCIPY (Jones et al. 2001).

\section{REFERENCES}

Abadi M. G., Navarro J. F., Steinmetz M., 2006, MNRAS, 365, 747

Allgood B., Flores R. A., Primack J. R., Kravtsov A. V., Wechsler R. H., Faltenbacher A., Bullock J. S., 2006, MNRAS, 367, 1781

An J., Evans N. W., Deason A. J., 2012, MNRAS, 420, 2562

Bailin J., Steinmetz M., 2005, ApJ, 627, 647

Battaglia G. et al., 2005, MNRAS, 364, 433

Battaglia G., Helmi A., Breddels M., 2013, New A Rev., 57, 52

Bell E. F. et al., 2008, ApJ, 680, 295

Belokurov V. et al., 2006, ApJ, 642, L137

Besla G., Kallivayalil N., Hernquist L., Robertson B., Cox T. J., van der Marel R. P., Alcock C., 2007, ApJ, 668, 949

Binney J., 2013, New A Rev., 57, 29

Binney J., Piffl T., 2015, MNRAS, 454, 3653

Binney J. J., Davies R. L., Illingworth G. D., 1990, ApJ, 361, 78

Biviano A., Girardi M., 2003, ApJ, 585, 205

Bland-Hawthorn J., Gerhard O., 2016, ARA\&A, 54, 529

Brown A. G. A., Velázquez H. M., Aguilar L. A., 2005, MNRAS, 359, 1287

Bullock J. S., Johnston K. V., 2005, ApJ, 635, 931 (BJ05)

Candlish G. N., Smith R., Moni Bidin C., Gibson B. K., 2016, MNRAS, 456,3456

Cappellari M., 2008, MNRAS, 390, 71

Carlberg R. G. et al., 1997a, ApJ, 476, L7

Carlberg R. G. et al., 1997b, ApJ, 485, L13

Cooper A. P. et al., 2010, MNRAS, 406, 744

Cooper A. P., Cole S., Frenk C. S., Helmi A., 2011, MNRAS, 417, 2206

Côté P. et al., 2001, ApJ, 559, 828

Courteau S. et al., 2014, Rev. Mod. Phys., 86, 47

De Lucia G., Helmi A., 2008, MNRAS, 391, 14

De Silva G. M. et al., 2015, MNRAS, 449, 2604

Deason A. J., Belokurov V., Evans N. W., An J., 2012, MNRAS, 424, L44 
Deg N., Widrow L., 2013, MNRAS, 428, 912

Dehnen W., Binney J., 1998, MNRAS, 294, 429

Deng L.-C. et al., 2012, Res. Astron. Astrophys.12, 735

Diakogiannis F. I., Lewis G. F., Ibata R. A., 2014, MNRAS, 437, 3172

Diakogiannis F. I., Lewis G. F., Ibata R. A., Guglielmo M., Kafle P. R., Wilkinson M. I., Power C., 2017, MNRAS, 470, 2034

Duarte M., Mamon G. A., 2015, MNRAS, 453, 3848

Dubinski J., Carlberg R. G., 1991, ApJ, 378, 496

Eadie G. M., Harris W. E., 2016, ApJ, 829, 108

Elahi P. J., Thacker R. J., Widrow L. M., 2011, MNRAS, 418, 320

Elahi P. J., Welker C., Power C., Lagos C. d. P., Robotham A., Cañas R., Poulton R., 2017, MNRAS, in press

Faltenbacher A., Mathews W. G., 2007, MNRAS, 375, 313

Fattahi A. et al., 2016, MNRAS, 457, 844

Fellhauer M. et al., 2006, ApJ, 651, 167

Freeman K., Bland-Hawthorn J., 2002, ARA\&A, 40, 487

Gaia Collaboration et al., 2016, A\&A, 595, A2

Gebhardt K., Fischer P., 1995, AJ, 109, 209

Gilmore G., Wilkinson M. I., Wyse R. F. G., Kleyna J. T., Koch A., Evans N. W., Grebel E. K., 2007, ApJ, 663, 948

Gnedin O. Y., Brown W. R., Geller M. J., Kenyon S. J., 2010, ApJ, 720, L108

Gustafsson M., Fairbairn M., Sommer-Larsen J., 2006, Phys. Rev. D, 74, 123522

Helmi A., 2004, MNRAS, 351, 643

Helmi A., 2008, A\&A Rev., 15, 145

Hernquist L., 1990, ApJ, 356, 359

Hunter J. D., 2007, Comput. Sci. Eng., 9, 90

Ibata R., Lewis G. F., Irwin M., Totten E., Quinn T., 2001, ApJ, 551, 294

Irrgang A., Wilcox B., Tucker E., Schiefelbein L., 2013, A\&A, 549, A137

Jeans J. H., 1915, MNRAS, 76, 70

Jing Y. P., Suto Y., 2002, ApJ, 574, 538

Johnston K. V., Bullock J. S., Sharma S., Font A., Robertson B. E., Leitner S. N., 2008, ApJ, 689, 936 (J08)

Jones E. et al., 2001, SciPy: Open source scientific tools for Python, available at: http://www.scipy.org/

Kafle P. R., Sharma S., Lewis G. F., Bland-Hawthorn J., 2012, ApJ, 761, 98

Kafle P. R., Sharma S., Lewis G. F., Bland-Hawthorn J., 2014, ApJ, 794, 59

Kazantzidis S., Kravtsov A. V., Zentner A. R., Allgood B., Nagai D., Moore B., 2004, ApJ, 611, L73

Kazantzidis S., Abadi M. G., Navarro J. F., 2010, ApJ, 720, L62

King III C., Brown W. R., Geller M. J., Kenyon S. J., 2015, ApJ, 813, 89

Kipper R., Tenjes P., Tihhonova O., Tamm A., Tempel E., 2016, MNRAS, 460,2720

Law D. R., Majewski S. R., Johnston K. V., 2009, ApJ, 703, L67

Li Y.-S., White S. D. M., 2008, MNRAS, 384, 1459

Lin D. N. C., Lynden-Bell D., 1982, MNRAS, 198, 707

Łokas E. L., 2009, MNRAS, 394, L102

McConnachie A. W. et al., 2009, Nature, 461, 66

McKinney W., 2012, Python for Data Analysis: Data Wrangling with Pandas, NumPy, and IPython. O'Reilly Media, Inc.

McLaughlin D. E., 1999, ApJ, 512, L9

McMillan P. J., 2011, MNRAS, 414, 2446

Majewski S. R., 1993, ARA\&A, 31, 575

Marigo P., Girardi L., Bressan A., Groenewegen M. A. T., Silva L., Granato G. L., 2008, A\&A, 482, 883

Martell S. L. et al., 2017, MNRAS, 465, 3203

Miyamoto M., Nagai R., 1975, PASJ, 27, 533

Navarro J. F., Frenk C. S., White S. D. M., 1996, ApJ, 462, 563

Pérez F., Granger B. E., 2007, Comput. Sci. Eng., 9, 21

Perryman M. A. C., 2002, Ap\&SS, 280, 1

Piffl T. et al., 2014, A\&A, 562, A91

Price-Whelan A. M., Johnston K. V., 2013, ApJ, 778, L12

Rashkov V., Pillepich A., Deason A. J., Madau P., Rockosi C. M., Guedes J., Mayer L., 2013, ApJ, 773, L32
Read J. I., 2014, J. Phys. G Nuclear Phys., 41, 063101

Richardson T., Fairbairn M., 2013, MNRAS, 432, 3361

Samurović S., Lalović A., 2011, A\&A, 531, A82

Sanders J. L., Binney J., 2013, MNRAS, 433, 1826

Sanderson R. E., Helmi A., Hogg D. W., 2015, ApJ, 801, 98

Sawala T. et al., 2016, MNRAS, 457, 1931

Sharma S., Johnston K. V., 2009, ApJ, 703, 1061

Sharma S., Johnston K. V., Majewski S. R., Bullock J., Muñoz R. R., 2011a, ApJ, 728, 106

Sharma S., Bland-Hawthorn J., Johnston K. V., Binney J., 2011b, ApJ, 730, 3

Smith M. C. et al., 2007, MNRAS, 379, 755

Springel V. et al., 2008, MNRAS, 391, 1685

Starkenburg E. et al., 2009, ApJ, 698, 567

Tonry J. L., 1983, ApJ, 266, 58

van der Walt S., Colbert S. C., Varoquaux G., 2011, Computing in Science \& Engineering, 13

Vera-Ciro C. A., Sales L. V., Helmi A., Navarro J. F., 2014, MNRAS, 439, 2863

Vogelsberger M. et al., 2014, MNRAS, 444, 1518

Walker M. G., Mateo M., Olszewski E. W., Peñarrubia J., Wyn Evans N., Gilmore G., 2009, ApJ, 704, 1274

Wang W., Han J., Cooper A. P., Cole S., Frenk C., Lowing B., 2015, MNRAS, 453,377

Waskom M. et al., 2014, Seaborn: v0.5.0, available at: https://doi. org/10.5281/zenodo. 12710

Wilkinson M. I., Evans N. W., 1999, MNRAS, 310, 645

Wolf J., Martinez G. D., Bullock J. S., Kaplinghat M., Geha M., Muñoz R. R., Simon J. D., Avedo F. F., 2010, MNRAS, 406, 1220

Xue X. X. et al., 2008, ApJ, 684, 1143

Yanny B. et al., 2009, AJ, 137, 4377

Zolotov A., Willman B., Brooks A. M., Governato F., Brook C. B., Hogg D. W., Quinn T., Stinson G., 2009, ApJ, 702, 1058

\section{APPENDIX}

\section{The case of accreted DM tracer populations}

Here, we demonstrate key results obtained using the accreted DM tracer populations although this may not have any observational relevance as it is not possible to directly observe the DM. None the less, we conduct the exercise to establish a few point discussed below. First, in Fig. A1 (analogous to Fig. 4), we demonstrate key spatio-kinematic properties of the accreted DM tracer populations (with sample size of $10^{5}$ ) from BJ05 and J08 simulations. We note that similar to Fig. 4, where stellar tracer populations are utilized, both the $\sigma_{r}$ and $\sigma_{\theta}$ (also $\sigma_{\phi}$, not shown in the figure) attain highest value for small $r$ and vice-versa, which turn-over at $r \simeq 5 \mathrm{kpc}$. Similarly, except the case of $\operatorname{circ}$ halo, all the simulated stellar halo have predominantly radial orbits. However, we note that compared to the earlier case when stellar tracers are used, the velocity dispersion profiles and, hence, also anisotropy, have larger inter-halo scatter. Notably, we also find that the density slope at $r<$ break-radius is comparatively steeper in the current case. Secondly, the corresponding reconstructed mass profiles of the individual haloes are already shown with blue lines in Fig. 5. Finally, in Fig. A2 (analogous to Fig. 6c), we present the distributions of the biases in the estimated masses at various distance range.

Number of DM tracers we utilize in Fig. 5 are roughly of the similar order of magnitude to that of the stellar tracers we had in Section 4 , i.e. $10^{5}$. We find that in this case, the overall mass offset is $\sim 3$ per cent with $\sim 7$ per cent $(\sim 6$ per cent when corrected for random uncertainty) of dispersion, which are clearly better than the respective errors of 12 per cent and 14 per cent we obtained earlier with the stellar tracer populations. This is expected and has a 

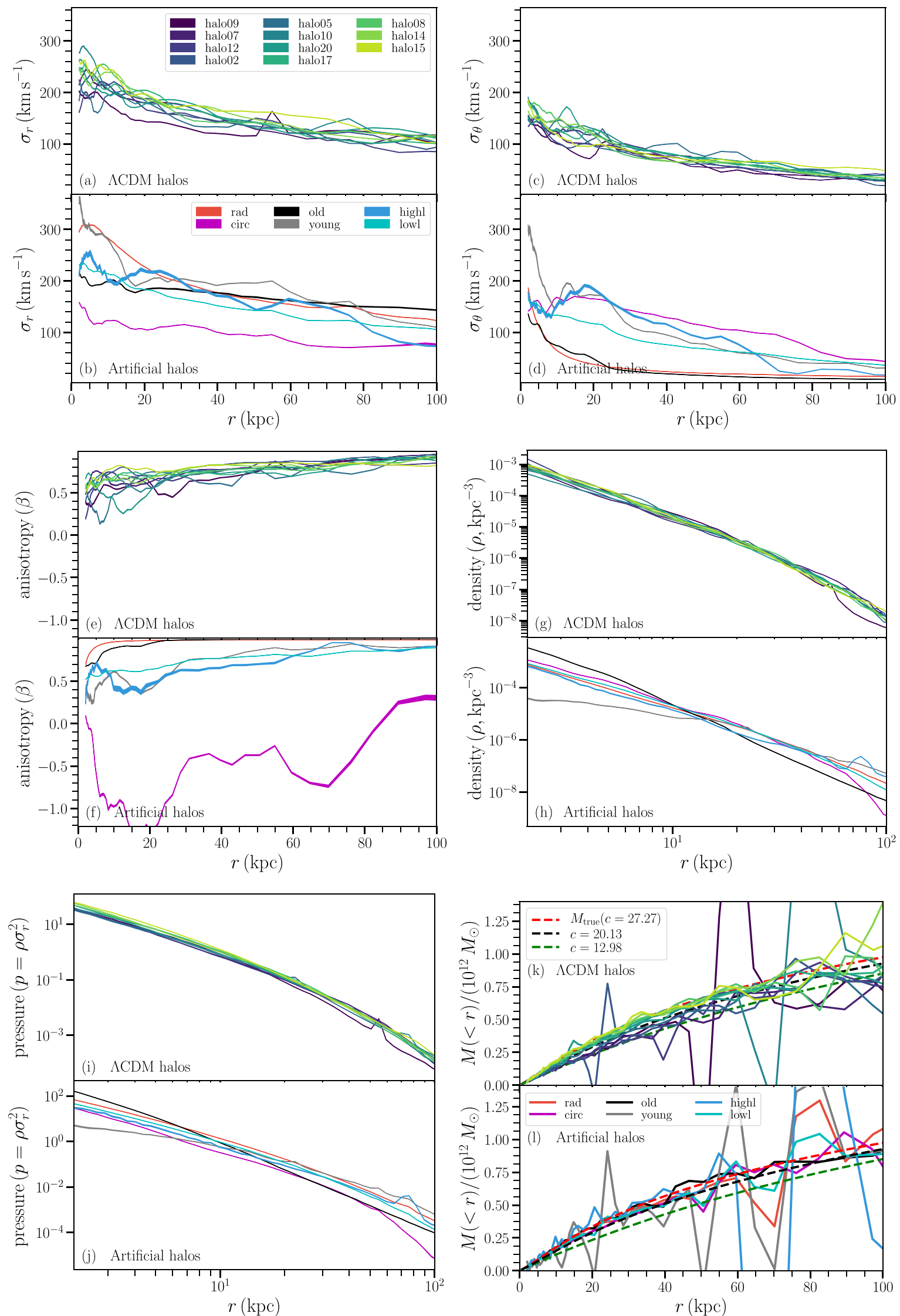

Figure A1. Analogous to Fig. 4, but for the case when accreted DM particles taken from BJ05 and J08 simulations are used as a dynamical tracer. 


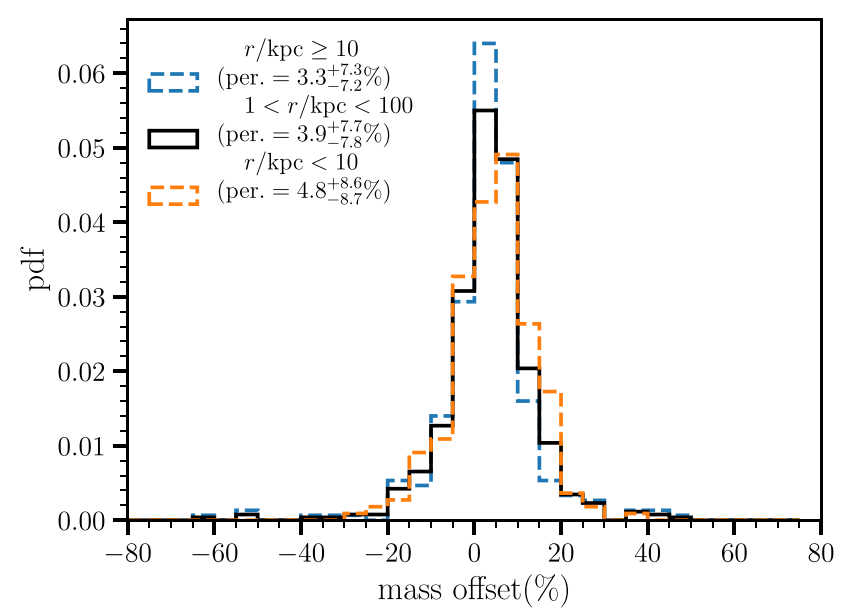

Figure A2. Error analysis of the estimated masses of the parent galaxy using the accreted DM tracer populations of the $11 \Lambda \mathrm{CDM}$ haloes, with labels same as Fig. 6(c).

physical cause. As mentioned in Section 2, in the BJ05 simulation, the stars are 'painted on' by assigning a luminosity weight to each dark matter particle within an accreted satellite. These luminosity weights are proportional to the binding energy of the satellite, meaning the stellar tracer populations generally come from the core of the accreted satellites; hence, it takes longer to relax compared to the DM tracer populations, resulting larger upset in the estimated mass profiles.

This paper has been typeset from a $\mathrm{T}_{\mathrm{E}} \mathrm{X} / \mathrm{L} \mathrm{T} \mathrm{E} \mathrm{X}$ file prepared by the author. 